\title{
INDICADORES DE BIENESTAR PARA PUEBLOS \\ TRADICIONALES - IBPT: VÁLIDOS PARA EVALUAR IMPACTOS DEL CAMBIO CLIMÁTICO EN AMAZONIA
}

\author{
Indicadores de bem-estar para povos tradicionais - IBPT: válidos para \\ avaliar os impactos das mudanças climáticas na Amazônia
}

\author{
Luis Eduardo Acosta Muñoz \\ Economista; Especialista en proyectos de desarrollo; \\ M.Sc. en desarrollo sostenible de sistemas agrarios; \\ Ph.D. en desarrollo, globalización y cooperación \\ internacional. Investigador Principal. Instituto \\ Amazónico de Investigaciones Científicas, Sinchi. \\ Colombia. \\ Correo electrónico: lacosta@sinchi.org.co \\ Informações do artigo \\ Recebido em: 13/02/2017 \\ Aceito em: 04/05/2017
}

\begin{abstract}
Resumen
El presente artículo expone los antecedentes de los que hoy se conoce como el paradigma de los impactos del cambio climático en los sistemas naturales y humanos; en particular el rigor de sus impactos en la Amazonia; se resalta el papel de los conocimientos tradicionales asociados a la biodiversidad, sus experiencias y cosmogonías, como una alternativa encaminada no solo para entender los rigores del cambio climático, sino las experiencias que se pueden retomar como propuestas a la mitigación del cambio climático. Presenta una propuesta que se encamina a la obtención de información en la Amazonia, mediante la cual se pretende contribuir a ampliar las evaluaciones de los impactos del cambio climático sobre las estructuras sociales con un enfoque metodológico diferencial.

Palabras clave: Cambio climático. Indicadores. Pueblos Tradicionales. Indígenas. Amazonia.
\end{abstract}

\section{Introducción}

En los albores del siglo XXI y a nivel planetario, se percibe con mayor certeza y claridad la existencia de un paradigma que expone los rigores de los impactos del cambio climático sobre los ecosistemas y las sociedades que los habitan. Denota la vigencia del modelo económico de crecimiento y las mayores intervenciones humanas sobre el medio ambiente, que convergen a generar mayor incertidumbre y vulnerabilidad a la sostenibilidad de los sistemas humanos y naturales, en razón a la tendencia hacia una mayor intensidad de los impactos.

En general los estudios para evaluar los impactos del cambio climático sobre los ecosistemas, son más recurrentes y cada vez es mayor el número de documentos que abordan esa preocupación mundial. Por el contrario, las evaluaciones sobre los impactos en las estructuras sociales y culturales son, aún, muy incipientes máxime cuando se trata de las sociedades tradicionales que habitan los ecosistemas de los bosques húmedos tropicales en la Amazonia. 
Como se sabe, la Amazonia es una región que se distingue por contar: con la mayor cobertura de la tierra en bosques húmedos tropicales y cuerpos de agua, con una superficie de $7.413 .827 \mathrm{~km}^{2}$, que comparten ocho países; una biodiversidad de especies florísticas, faunísticas, ícticas; una diversidad cultural de más de 420 pueblos indígenas diferenciados; sus ecosistemas ofrecen una gama de servicios ecosistémicos que aportan a generar un alto valor en el equilibrio hídrico global y continental. Existen ciertas dinámicas socioeconómicas que han incentivado la ocupación y la intervención, conllevando a intensificar los procesos de deforestación y la disminución de la biodiversidad, produciendo la pérdida de hábitats y la fragmentación de los ecosistemas, con importantes procesos de urbanización no planificados. Lo anterior denota, no solo una creciente degradación ambiental que está alterando los servicios ecosistémicos amazónicos, sino la seguridad hídrica, energética, económica, alimentaria y de la salud de los habitantes, afectadas las relaciones sociales y generado un número creciente de situaciones de conflicto por la apropiación indebida de los recursos naturales, entre otros. Ese conjunto de situaciones ha implicado una tendencia al aumento de la vulnerabilidad frente a las inundaciones, las sequías, debidas al cambio climático (OTCA, PNUMA, 2009, p. 21-27).

Lo anterior, reviste importancia en razón de la vinculación de las sociedades tradicionales con la sociedad nacional, y su participación en las economías locales y el estado, que se ha caracterizado por generar procesos de empobrecimiento, pérdida de cohesión social, exclusión y vulnerabilidad social, dependencia alimentaria por la disminución de variabilidad genética en sus espacios de cultivo; degradación de los mecanismos de transmisión de los conocimientos tradicionales asociados a la biodiversidad a las nuevas generaciones, entre otros. Es un contexto que no ha contado con enfoques conceptuales y metodológicos fiables y rigurosos, que permitan sostener procesos interculturales por diferentes actores institucionales encaminados a afianzar la sostenibilidad de los modos de vida y territorios de dichas sociedades tradicionales, y por ente en la protección de sus conocimientos tradicionales asociados a la biodiversidad.

El presente artículo expone en primer lugar los antecedentes de los que hoy se conoce como el paradigma de los impactos del cambio climático en los sistemas naturales y humano; en particular el rigor de sus impactos en la Amazonia; en segundo lugar, se resalta el papel de los conocimientos tradicionales asociados a la biodiversidad, sus experiencias y cosmogonías, como una alternativa encaminada no solo para entender los rigores del cambio 
climático, sino las experiencias que se pueden retomar como propuestas a la mitigación del cambio climático. En tercer lugar, se presenta una propuesta que se encamina a la obtención de información en la Amazonia, mediante la cual se pretende contribuir a ampliar las evaluaciones de los impactos del cambio climático sobre las estructuras sociales con un enfoque metodológico diferencial.

En ese sentido, Acosta (2013), generó la identificación y diseño de Indicadores de Bienestar Humano Indígena (IBHI), los cuales se fundamentan en los aspectos conceptuales del bienestar humano en términos de la abundancia de los pueblos indígenas de la Amazonia colombiana y se enmarcan en el enfoque de las capacidades: control colectivo del territorio; agencia cultural autónoma; garantizar la autonomía alimentaria; lograr un ambiente tranquilo; el auto-cuidado y la reproducción. Es una propuesta que responde a las exigencias antes señaladas, con la participación comunitaria en la vigilancia de los usos futuros que la información pueda tener; y que permita contribuir a retroalimentar la conformación de las políticas públicas más acordes con las particularidades sociales, culturales y territoriales de las sociedades indígenas.

La aplicación de los IBHI y los resultados logrados en la Amazonia colombiana, permitió generar una experiencia piloto de aplicación y análisis en la frontera que comparten Brasil y Colombia; fruto de ese proceso, los indicadores de bienestar humano se denominaron Indicadores de Bienestar Humano para Pueblos Tradicionales (BIPT), ampliando y con las debidas adaptaciones, las posibilidades de su aplicación en diversas poblaciones tradicionales de la Amazonia brasilera.

La conceptualización y aplicación de los IBPT en la Amazonia es una propuesta que contribuye no solo a instrumentalizar los derechos humanos de las sociedades tradicionales reconocidos por la ONU (2007), sino a contar con una información de carácter holístico que referencia los impactos no solo de su vinculación con la sociedad y las economías locales, sino los impactos del cambio climático sobre sus estructuras sociales, culturales y de gobernabilidad. Estos resultados abren un camino importante para contar con propuestas metodológicas que sirvan para adelantar evaluaciones justas y fiables de la realidad de las sociedades amazónicas; y abren un espacio para que las entidades públicas y ONG, se comprometan y apoyen la sostenibilidad de los procesos en marcha. 


\section{El paradigma de los impactos del cambio climático}

En la última década y a nivel global se viene alertando, y cada vez más con mayor consenso, sobre la existencia de un cambio en el clima y sus impactos en los ecosistemas y en la vida de las sociedades que los habitan. Sostienen que los impactos ocasionados por los diferentes fenómenos como altas temperaturas, sequías, inundaciones, ciclones e incendios forestales, muestran que los ecosistemas y los sistemas humanos son altamente vulnerables a la actual variabilidad climática. Y que los impactos no solo generan la alteración de los ecosistemas, sino que son severamente negativos para las personas que viven en la pobreza cuyos impactos se presentan sobre los medios de subsistencia, reducciones en los rendimientos de los cultivos o destrucción de sus hogares; y que generalmente ocasionan escases de alimentos con los consecuentes aumentos en los precios y por ende a una mayor inseguridad alimentaria (IPCC, 2014).

Una de las conclusiones del Panel Intergubernamental sobre Cambio Climático (IPCC, 2014, p. 4) es que las evaluaciones a los ecosistemas naturales son más recurrentes y concluyentes, que aquellas practicadas a los sistemas humanos, que hoy se sabe, son especialmente sensibles y vulnerables a las respuestas de adaptación y mitigación en razón a la multiplicidad de causas que lo ocasionan. Los autores referenciados señalan, que son producto de procesos sociales interrelacionados que generan desigualdades socioeconómicas, discriminación por motivo de género, clase, etnicidad, edad, entre otras. De otro lado, el Grupo Semillas (2014, p.3-4), y Ulloa, Escobar, Donato, Escobar, (2008: 19 21), coinciden en relacionar la existencia de diferentes fenómenos de carácter socioeconómico relacionados en profundizar los impactos del cambio climático en las estructuras sociales y culturales, a saber:

a) Las cadenas de valor industriales de alimentos ocasionan entre el 15 y el $18 \%$ de las emisiones globales de gases con efecto de invernadero (GEI), las cuales son producidas por el cambio en el uso del suelo y la deforestación ocasionada por la agricultura y las cadenas de valor industriales.

b) América Latina, aporta solamente un $6 \%$ de las emisiones de GEI, sufriendo fuertes impactos en sus ecosistemas y recursos debidos al cambio climático. Se señala que los principales impactos y riesgos para la región son: aumento de eventos climáticos extremos, transformaciones en la Amazonia, pérdida de 
bosques por nuevos usos de la tierra, pérdida de biodiversidad, desaparición de los páramos y retroceso de los glaciares, aumento de las enfermedades tropicales, y vulnerabilidad en las fuentes de energía (hidroeléctrica), entre otros.

c) La expansión de la frontera agrícola es motivada por la deforestación, con una participación entre el 70 y el $90 \%$ de la misma a nivel global. Al respecto, en el año 2005, la deforestación acumulada en la Amazonía alcanzó la cifra de 857.666 km2, lo que significó que a lo largo del tiempo la cobertura vegetal de la región se haya reducido aproximadamente en un 17\%. De prevalecer esa tendencia, y si la pérdida del bosque de la Amazonia supera el 30\%, se pronostica que se reducirá la liberación de vapor de agua y por ende la disminución de las precipitaciones; y que en razón al volumen de agua que drena hacia el océano Atlántico (15 y 20\% de la descarga total mundial de agua dulce fluvial), podría influir en modificar los ciclos hídricos amazónicos con un potencial para influir sobre algunas de las grandes corrientes oceánicas, que son importantes reguladoras del sistema climático global. (OTCA, PNUMA (2009:15-17).

d) La existencia de actores que ejercen control y en particular de territorios de sociedades tradicionales, motivados por la posibilidad de apropiación de recursos naturales que aporten al crecimiento económico. Las alteraciones en dichas sociedades y territorios tienen gran impacto, dado que pueden llevar a conflictos y expulsión de poblaciones en los territorios.

e) La implementación de proyectos y megaproyectos (generación y transporte energético, industrias extractivas, vías de comunicación), afectan ambiental y culturalmente sus territorios, e inciden en las prácticas ancestrales de manejo de los recursos. Las estrategias que buscan mitigar el cambio climático, generan también impactos negativos sobre los territorios de las sociedades tradicionales.

f) Los impactos y el aumento de la temperatura ambiental, impactan igualmente el bienestar humano de la población y sus asentamientos; se asocian, con el incremento de enfermedades como malaria, dengue, fiebre amarilla, con importantes consecuencias para la salud mental, generalmente en asentamientos que no cuentan con la prestación de servicios públicos. 
En relación con la Amazonia, según OTCA, PNUMA (2009, p.216-277), sostienen que el bosque amazónico está íntimamente relacionado con el clima a nivel mundial. Que esta región influye sobre el clima actuando como un gigantesco consumidor de calor, y absorbe la mitad de la energía solar que le llega mediante la evaporación del agua de su follaje; es una reserva de carbono la cual, y dependiendo de la intensidad de los procesos de poblamiento e intervención, es susceptible de ser liberado a la atmósfera en razón a la deforestación, los incendios y las sequías, lo que contribuye al calentamiento global.

Afirman OTCA, PNUMA (2009), Mardas, Bellfield, Jarvis, Navarrete, Comberti, (2013, p.3) que los recursos naturales de la Amazonia son la base de la seguridad hídrica, energética, alimentaria y de la salud, para la población y la economía a nivel regional. Enfatizan, que el desarrollo económico en la Amazonia que se ha basado en la deforestación, compromete la estabilidad de los ecosistemas, amenazando el bienestar y los derechos de la población; y que el cambio climático multiplicará esas amenazas a medida que la temperatura aumente, los patrones de precipitación varíen y los fenómenos extremos sean cada vez más frecuentes e intensos.

La existencia de fenómenos socioeconómicos como la empradización que amplía la frontera agrícola, las economías extractivas de los recursos naturales, la implementación de grandes megaproyectos de infraestructura, entre otros, que guardan una relación con la mayor intensidad de los impactos del cambio del clima sobre los ecosistemas y en los asentamientos humanos, en la región amazónica; se podría inferir que éstos son de mayor drasticidad en relación con las sociedades tradicionales, sus modos de vida y territorios, que dependen de los recursos de la biodiversidad, y que cosmogónicamente guardan una íntima relación con la naturaleza en la Amazonia. Se entiende esa relación, como la vigencia de una práctica espiritual lograda históricamente y se expresa en un conjunto de valores, conocimientos, tecnologías e innovaciones, asociadas a la biodiversidad. Donde sus modos de vida se fundamentan en los saberes tradicionales que permite un conocimiento para el uso y manejo de la biodiversidad que les garantiza la sostenibilidad de la agricultura, la cacería, la pesca, la recolección y transformación de productos silvestres, la medicina natural. Acosta et al (2011), Gasche y Vela (2004), Gasche (2012), Acosta (2013), Uribe (2015, p. 29-30).

Según Feldt (2011, p.01), estima que en razón de los impactos del cambio climático aludidos, en las sociedades tradicionales conllevará forzosamente a modificar sus estructuras tradicionales, costumbres de vida y de alimentación, como consecuencia de las alteraciones 
de la relación sociedad - naturaleza; por su parte Ulloa, Escobar, Donato, Escobar, (2008, p. 18), aseguran, que no solo se estima la pérdida de la biodiversidad, sino la degradación de sus condiciones de autonomía; se inducirán desequilibrios tanto en los comportamientos comunitarios como en los espacios de participación, con una tendencia a la pérdida del reconocimientos de la autoridad tradicional; situación que podría ser más intensa, en razón a las sociedades tradicionales mantienen una vinculación con las economías locales, que contribuye a la degradación de los conocimientos tradicionales asociados a la biodiversidad. (Acosta, 2013).

\section{La adaptación y mitigación al cambio climático: un camino por construir}

La adaptación y mitigación son dos conceptos que guardan una correspondencia con la prevención de fenómenos y sus impactos sean estos de carácter ambiental y/o socioeconómicos. La primera se refiere al proceso que un individuo y/o una sociedad construye para modificar sus comportamientos, hábitos, costumbres, para amoldarse a las condiciones, normas y reglas, del medio donde se desenvuelve, que les permitan enfrentar los impactos de diferentes fenómenos sean estos de tipo ambiental y/o socioeconómico; en otros términos, son las actividades realizadas por los miembros de una sociedad para evitar, resistir o aprovechar la variabilidad, los cambios y los efectos del clima actuales o pronosticados, que permitan disminuir la vulnerabilidad y aumentar la capacidad de respuesta.

La segunda consiste en diferentes medidas que se ejecutan y anticipan frente a un proceso de posibles desastres, con el fin de frenar en lo posible la escalada de la vulnerabilidad y aminorar el impacto del mismo. Según UNDP-DHA (1994) generalmente se asocia a las medidas para minimizar el impacto de un desastre, pero ejecutables en todo momento; ayudando a la gente a sobrevivir, a preservar sus sistemas de sustento y a poder afrontar mejor los desastres futuros. Tiene un ámbito de la gestión del Estado, en razón a que las medidas de mitigación como son de largo plazo, deben ser contempladas en los instrumentos de planeación nacionales y a nivel local.

En general son procesos que requieren, no solo de estudios de carácter holístico, sino de procesos de participación para que las tomas de decisiones sean mutuamente corresponsables entre la sociedad y del Estado. 
De acuerdo con Elías y Cardona (2015), los procesos de mitigación a pesar de los esfuerzos globales, acordados en las negociaciones internacionales auspiciadas en el seno de la Convención Marco de la Naciones Unidas sobre Cambio Climático (CMNUCC) para disminuir las emisiones de gases de efecto invernadero causantes del calentamiento global, son insuficientes y han privilegiado enfoques técnicos centralistas y economicistas, para mitigar la tendencia del cambio climático. Que en ese contexto es necesario que la población se adapte a las nuevas condiciones climáticas, e implica reducir la vulnerabilidad social, ambiental y económica, adaptar las prácticas productivas a las nuevas condiciones y fortalecer sus capacidades de resiliencia.

Los autores referenciados señalan, que el énfasis de los esfuerzos de mitigación ha estado centrado en reducir las emisiones por deforestación y degradación a través de proyectos REDD y REDD+; sin embargo, presentan la particularidad que en dichas iniciativas se involucran solamente a los países en desarrollo donde ocurren las mayores tasas de deforestación, enfatizando un llamado para que los países industrializados promuevan sus propios esfuerzos para reducir sus emisiones que como se sabe provienen del alto consumo de hidrocarburos.

En su momento Bergkamp, Orlando, Burton, (2003) frente a existencia de los riesgos e incertidumbres que provienen del cambio climático sobre los recursos hídricos sostenían que las soluciones técnicas en la gestión de los mismos han resultado ser insuficientes; sostienen que no es suficiente el pronunciamiento de los expertos sino una participación más amplia de las partes interesadas y transparencia para desarrollar apoyo político en favor de compartir la carga y los beneficios de los impactos del cambio climático. Y que parte fundamental de ese logro reside en el "aprendizaje social". Esto último se entiende, como los procesos de participación necesarios de emprender con las sociedades tradicionales que habitan importantes zonas de biodiversidad; en particular, aquellas que se localizan en la Amazonia, que como se sabe, cuentan con los conocimientos que les ha permitido usar y manejar sosteniblemente los recursos naturales y que son fundamentales en el sostenimiento de sus modos de vida y bienestar. 


\section{Los conocimientos tradicionales y su papel en la mitigación del cambio climático}

Siguiendo a Elías y Cardona (2015), se comparte que los conocimientos tradicionales son fundamentales para entender, proteger y conservar los ecosistemas, y en particular para jugar un papel que permita disminuir las vulnerabilidades y prepararse ante las contingencias del cambio climático que afectará los modos de vida en sus territorios. Afirman dichos autores, que los conocimientos tradicionales, en tanto recursos colectivos, son fundamentales para enfrentar el cambio climático y pueden constituirse en el mecanismo que vincule el capital social territorial, ya que conlleva al fortalecimiento de la cooperación, la solidaridad y la reciprocidad entre personas y comunidades. De otra parte Acosta y Mendoza (2006:115) indican que los conocimientos tradicionales asociados a la biodiversidad son claves en la construcción del desarrollo sostenible en la Amazonia colombiana; y que en esa dirección, era necesario profundizar en el conocimiento hasta ahora alcanzado, para permitir dimensionar los sistemas de producción indígenas y no indígenas como un complejo de interrelaciones entre el ser humano, el medio ambiente y los procesos productivos; y prioritario adelantar un análisis sobre los cambios culturales y sociales generados en razón a su vinculación con la sociedad nacional y las economías locales.

$Y$ ese proceso permitió reconocer que era necesario adoptar como estrategia metodológica el apoyo a los procesos gestados por los pueblos indígenas, en razón a sus demandas generadas por los planes de vida, y en el diálogo y concertación con los pueblos indígenas a través del consentimiento previo informado, como figura jurídica para reglamentar el acceso a conocimientos tradicionales y la repartición de beneficios en sus territorios.

Recientes resultados de investigación adelantados en la Amazonia, particularmente en la triple frontera que comparten Colombia, Brasil y Perú, señalan el interés no solo de los organismos del Estado sino de las sociedades tradicionales por revalorar los conocimientos tradicionales, y se demuestra que los mismos tienen un valor fundamental en la búsqueda de respuestas para enfrentar los desafíos del cambio climático. Las sociedades tradicionales que se localizan en la región, han desarrollado prácticas productivas y estilos de vida que pueden ser interesantes para la mitigación (Acosta, 2013; De la Cruz, 2015, De la Cruz, 2016; Lacerda, 2016). Al respecto Ulloa, Escobar, Donato, Escobar, (2008: 18), concluyen que cada cultura tiene sus propias concepciones, relaciones y percepciones sobre la naturaleza y sus 
territorios, al igual que sobre la historia de los cambios ambientales. Los cambios climáticos se han dado históricamente y los pueblos indígenas han generado diferentes estrategias para enfrentarlos. Dentro de esas, la generación de alternativas de producción en diversos ecosistemas, el establecimiento de redes de intercambio con otros pueblos, con miras a confrontar las transformaciones ambientales.

Sin embargo, en la Amazonia y de acuerdo con Lacerda (2016), las políticas públicas y su práctica, por más que existen las mayores voluntades, su aplicación se realiza sobre realidades históricas que no han permitido la participación de la sociedad para que los esfuerzos estatales generen los mayores beneficios sociales. Y su implementación se realiza sin contar con las metodologías, ni con los procesos de análisis que permitan entender y dimensionar las situaciones sociales, culturales, económicas y políticas, de carácter histórico, que han impedido que las sociedades tradicionales hayan forjado su emancipación para alcanzar mayores posibilidades de bienestar humano por los esfuerzos del estado.

Frente a los procesos de adaptación y mitigación que exige los impactos del cambio climático en los territorios y modos de vida de las sociedades tradicionales que habitan importantes ecosistemas de la Amazonia; la armonización de esfuerzos entre las comunidades y las acciones del Estado, es una condición necesaria para afianzar procesos de adaptación y consolidar procesos de mitigación participativos, que permitan enfrentar mancomunadamente los rigores del cambio climático.

Al respecto Mardas, Bellfield, Jarvis, Navarrete, Comberti, (2013:15), afirman que el cambio climático brinda oportunidades para las instancias de toma de decisiones, en razón a las responsabilidades para lograr un balance adecuado entre desarrollo económico y la salvaguardia de los ecosistemas en la Amazonia; aspecto clave para lograr una seguridad sostenible y el bienestar de la población. En esa dirección proponen: un cambio en el paradigma para que se reconozca que los ecosistemas de la Amazonia no solo influyen en el cambio del clima a nivel mundial, sino que son una seguridad que permite sustentar el bienestar y la prosperidad de la sociedad a nivel regional; mayor conocimiento sobre los impactos y riesgos cuya información con la capacidad de retroalimentar los procesos de toma de decisiones, a través de un conjunto de herramientas de información y acciones de mitigación. Respecto de este último punto, recomiendan definir un conjunto de indicadores sociales, ambientales y económicos, que faciliten el monitoreo, intercambio de información sobre la seguridad hídrica, energética, alimentaria y de la salud, en la Amazonia. 
Por su parte, OTCA, PNUMA (2009:27) entre las estrategias que se plantean para enfrentar los impactos del cambio climático en la Amazonia, alientan en la necesidad de fortalecer los esfuerzos encaminados a la generación y difusión de información ambiental en la región, a través de un sistema de información ambiental amazónica que tenga en cuenta las plataformas existentes (sistemas georreferenciados, estadísticas, entre otros) y promover su articulación entre los ámbitos públicos y privados. En su oportunidad, Pardo (2007:14) enunciaba que era necesario avanzar en conformar enfoques integrales para entender los impactos del cambio global, que permitan emprender la comprensión de los factores sociales, para analizar cuáles son los efectos sobre la capacidad de funcionamiento de las sociedades, así como la relación entre ésta y la vulnerabilidad del sistema biofísico y su capacidad de adaptación.

\section{La creación y práctica de indicadores de bienestar humano: una alternativa para la evaluación de los modos de vida en las sociedades tradicionales}

La preocupación actual y en relación con los impactos del cambio climático, su incidencia en la sostenibilidad de los ecosistemas naturales y la población que los habita, exigen otras aproximaciones conceptuales y metodológicas de carácter multidimensional, que permita generar información acorde con las características y situaciones particulares de las sociedades y los ecosistemas en la Amazonia, que contribuya a plantear, desde la institucionalidad, acciones realistas y participativas -independientemente de la temática a tratar-, que involucren sus modos de vida y territorios. En ese sentido, Acosta (2013) adelantó una propuesta para la identificación y diseño de indicadores de bienestar humano, cuya metodología cuenta con la potencialidad de ser aplicada para entender los impactos del cambio climático en los territorios de las sociedades tradicionales en la Amazonia.

La propuesta se contextualiza en las iniciativas de diferentes organismos internacionales como la ONU, BM, BID, entre otros, que han generado procesos conceptuales y técnicos para conformar sistemas de indicadores para evaluar los alcances del desarrollo en territorios de sociedades tradicionales de países en vías de desarrollo. En general se reconoce que las cosmovisiones locales son fundamentales para evaluar tanto las políticas como las acciones de desarrollo y de bienestar en relación con las expectativas de vida y el respeto de las culturas tradicionales y al medio ambiente. Abren un espacio para una concepción propia 
del bienestar de los pueblos tradicionales, y la posibilidad de identificar y diseñar indicadores de carácter étnico y cultural, coherentes y fiables. (ONU, 2007; 2008).

Con el reconocimiento de los derechos humanos de los pueblos indígenas por parte de las Naciones Unidas (OIT, 2009), y diferentes encuentros a nivel internacional de los pueblos indígenas (Declaración de Política Kimberlee, Reunión de líderes indígenas de América Latina y el Caribe sobre los indicadores de bienestar humano (IBH), El Pacto de Pedregal realizado en México en 2004); la CEPAL y el Fondo Indígena desarrollaron un sistema de Indicadores Sociodemográficos de poblaciones y los Pueblos Indígenas de América Latina (SISPPI), contando como guía los siguientes ejes: distinguir a los pueblos indígenas del resto de la población a fin de establecer estadísticas descriptivas y referenciales, bajo el criterio de pertinencia cultural y que reconozcan los derechos colectivos de los pueblos indígenas logrados a nivel internacional (CELADE / CEPAL, 2007).

Estas experiencias históricas, apoyadas por las propias organizaciones indígenas, abrieron un espacio para que actores locales en conjunto con las poblaciones, desarrollaran indicadores específicos para la medición coherente de los modos de vida en diferentes territorios tradicionales. Acosta (2013) afirma que el contacto de los pueblos indígenas con la cultura occidental condujo, a lo largo de la historia, a una percepción errónea respecto de esas sociedades tradicionales, y revisando los índices de evaluación de diversos órganos de apoyo, control y fomento, concluye que a pesar de algunos avances todavía son ineficaces para la comprensión de las realidades tradicionales de la Amazonia.

El diseño de los IBHI pertinentes para evaluar el bienestar de los modos de vida de las sociedades indígenas en la Amazonia, se sustentó en la conformación de un modelo de análisis, que conjugó: 1) el marco holístico que define y explica el concepto de bienestar de los modos de vida indígena, que se sustenta a través de la abundancia; 2) el concepto de bienestar que define una relación sociedad - naturaleza y vista desde los antecedentes del desarrollo humano.

En primer lugar, el modelo se sustenta en el concepto de abundancia, que según Acosta (2013) se puede asumir como un concepto de bienestar, el cual hace parte de la cosmovisión de los pueblos indígenas en la Amazonia colombiana. Es una noción que encierra elementos diversos, que, a la vez, exige el apoyo de prácticas concretas de la vida ritual y cotidianas de la sociedad indígena. Esta noción se asocia con la noción moniyafue (Pueblos Murui - Muina) y que se traduce como la abundancia, la cual muestra el estado en el que se 
encuentra el ser humano en relación a uno o varios ecosistemas. Es un concepto de orden ritual en el que se conjugan una moral y una religiosidad propias. Se relaciona con el conocimiento de la palabra ritual de los ancianos como autoridades tradicionales de esta región (García, 2009; Acosta et al. (2011). En general, se asocia a esta noción la existencia de abundancia de comida y a la vez está ligada a connotaciones más amplias como es la de estar satisfecho. Se asocia con el concepto de vivir bien que es contar con un ambiente tranquilo que permita la vida en comunidad y contar con un ambiente apacible en los territorios indígenas, donde reinen: las buenas relaciones entre vecinos, relaciones intrafamiliares armónicas, participación de la vida comunitaria, respeto por los valores individuales y familiares, entre otros.

En segundo lugar, el modelo se sustenta sobre un conjunto de funcionamientos que definen las capacidades, a través de las cuales se pretende dar cuenta y evaluar el bienestar de los pueblos indígenas. En este modelo, el análisis de las capacidades se fundamenta sobre fenómenos de orden colectivo. De otro lado, los pueblos indígenas se hallan en contacto con las sociedades nacionales, el Estado y la economía regional. En estas condiciones, la intervención del Estado a través de instituciones como la escuela -educación elemental- y la salud -centros médicos y puestos de salud-, así como las intervenciones de la iglesia, debe contemplarse como parte de su realidad actual. Los efectos provocados por esos nuevos contactos en la consecución de los logros de bienestar son fundamentales. En ese sentido, avanzar en la construcción de un modelo conceptual que permita evaluar el bienestar humano en los pueblos indígenas en la Amazonia colombiana, implica identificar y determinar las capacidades asociadas a la noción de sostenibilidad de los modos de vida indígena, que como se viene sosteniendo se deben contextualizar en referencia del concepto de abundancia.

En esa dirección, Dubois (2008), Acosta, García, Dubois (2016) aportan a la conceptualización de un enfoque sobre las capacidades colectivas (ECC) que integran un modelo valido para evaluar el bienestar de los pueblos indígenas. Los autores consideran que el ECC propuesto, es idóneo para asumir la evaluación del bienestar humano de los pueblos indígenas en sus territorios; es un enfoque diferencial que permite la evaluación de: existencia de pluriactividades de autosuficiencia alimentaria como la agricultura, la cacería, la pesca, la recolección, la transformación; valores morales referidos las redes de solidaridad, la cooperación familiar, el respeto del principio de la reciprocidad y el reconocimiento de una 
autoridad tradicional, que conoce el medio ambiente y facilita los convenios con los seres de naturaleza para garantizar la vida. Es un modelo que reconoce como relevantes, la protección de todos los derechos humanos, las habilidades y las opciones de los individuos de ser capaces de satisfacer sus propias necesidades, la igualdad de oportunidades; la construcción de una equidad universal, la cual conlleve al mejoramiento de las oportunidades vitales para las generaciones actuales y futuras; sobre la base de una gobernabilidad, donde exista una sociedad civil fortalecida y participativa y unos gobiernos responsables.

Los autores señalados argumentan que el modelo conceptual para evaluar los modos de vida y territorios de los pueblos indígenas, se basa en las capacidades colectivas que guardan una correspondencia con el control y gestión de los territorios ancestrales en la figura del resguardo, que presentan un estado legal como propiedades privadas colectivas. Estas capacidades comprenden:

a) La capacidad de funcionamiento colectivo. Es contar con un gobierno propio e intercultural en los territorios sociedades tradicionales.

b) La capacidad de agencia cultural autónoma. Es tener control del territorio y de los inventarios Ecosistémicos; ser estables en el territorio; ser parte de un grupo tradicional autónomo y ser competente en la comunicación cultural e intercultural.

c) La capacidad para garantizar la autonomía alimentaria. Es tener la alimentación deseada, la seguridad de abastecimiento, y estar bien alimentado.

d) La capacidad para lograr un ambiente tranquilo. Es ser autónomo respecto de agentes y problemáticas sociales externas para el ejercicio de la territorialidad.

e) La capacidad para garantizar el auto-cuidado y reproducción. Ser personas saludables y tener un buen estado de salud.

A partir de cada una de estas capacidades y su conceptualización al igual que los respectivos funcionamientos que definen cada una, se identificaron, definieron y diseñaron los indicadores de bienestar para pueblos indígenas.

Los IBPT darán cuenta de las realidades y temáticas de importancia desde el punto de vista de los pueblos indígenas: la identidad, la espiritualidad, el conocimiento tradicional, las formas propias de organización social, los derechos colectivos y el patrimonio intangible. El 
monitoreo de los IBHI constituye una herramienta con la potencialidad de proveer información útil para visibilizar los modos de vida y territorios de los pueblos indígenas; y en particular, aportar información sobre los cambios que ocurren no solo por la vinculación a la sociedad y las economías locales, sino los generados por los cambios en el clima. La información que proveerán será un insumo para la formulación de políticas públicas, programas y acciones de gobierno, u otras instancias asociadas al desarrollo y al bienestar indígenas, que permitan que las familias indígenas no pierdan su autonomía e identidad que garantizan su bienestar, frente a los rigores e impactos generados por el cambio del clima.

En este sentido, la aplicación de los IBHI y los resultados logrados en la Amazonia colombiana, permitió generar una experiencia piloto de aplicación y análisis en la frontera que comparten Brasil y Colombia. Fruto del proceso de aplicación en la realidad de la frontera de Brasil, los indicadores de bienestar humano se denominaron Indicadores de Bienestar para los Pueblos Tradicionales (BIPT), ampliando y con las debidas adaptaciones, sus posibilidades de aplicación en diversas poblaciones tradicionales. La hipótesis inicial en la aplicación de los IBPT se fundamenta en la preocupación sobre la soberanía expresada por los pueblos tradicionales en sus propios territorios, asumiendo que frente a cualquier intervención sus valores y culturas deben ser percibidos como parte esencial en la planificación inicial hasta las evaluaciones finales.

En las tablas 2, 3, 4, 5 y 6, se presentan los IBPT cuyas conceptualizaciones muestran una propuesta con la potencialidad de visualizar los elementos de orden cualitativo y cuantitativo, sincrónico y diacrónico, de orden individual, social y colectivo, relacionados con las sociedades tradicionales y sus territorios en la Amazonia, con la potencialidad holística de mesurar las consecuencias que en el largo plazo el cambio climático y sus impactos tendrá sobre los ecosistemas y las sociedades que los habitan.

Tabla 2. IBHI de la capacidad de control colectivo del territorio

\begin{tabular}{|c|l|}
\hline \multicolumn{1}{|c|}{ Indicador } & Potencial de gobernabilidad en los territorios sociedades tradicionales \\
\hline \multirow{2}{*}{ Definición } & $\begin{array}{l}\text { Son las jerarquías básicas de gobierno propio e intercultural que existen para el } \\
\text { control social y político en los territorios sociedades tradicionales, y corresponden } \\
\text { a las categorías especiales de autoridad, sustentadas en cabeza de los caciques y } \\
\text { su espacio ritual en las malocas; los gobernadores y/o curacas de cabildos; } \\
\text { presidentes de asociaciones, entre otros. }\end{array}$ \\
\hline
\end{tabular}




\begin{tabular}{|l|l|}
\hline Pertinencia & $\begin{array}{l}\text { Las jerarquías básicas de gobierno propio e intercultural son un componente } \\
\text { fundamental del desarrollo humano autónomo en los territorios sociedades } \\
\text { tradicionales; conforman un modelo de gobierno y gobernabilidad que garantizan } \\
\text { el control social, político y ambiental en los territorios. }\end{array}$ \\
\hline Unidad de medida & $\begin{array}{l}\text { Número }\left(\mathrm{N}^{\circ}\right) \text { de autoridades tradicionales (i) en territorios sociedades tradicionales } \\
(\mathrm{j}) \text {, en el año }(\mathrm{k}) .\end{array}$ \\
\hline
\end{tabular}

Tabla 3. IBHI de la capacidad de control colectivo del territorio

\begin{tabular}{|l|l|}
\hline \multicolumn{1}{|c|}{ Indicador } & $\begin{array}{c}\text { Potencial de áreas con oferta de recursos naturales en territorios sociedades } \\
\text { tradicionales }\end{array}$ \\
\hline Definición & $\begin{array}{l}\text { Corresponde a la base natural biodiversa con que cuentan los territorios de } \\
\text { sociedades tradicionales; incumbe todos los activos que conforman la oferta de } \\
\text { recursos naturales disponibles, a partir de los cuales se sostienen los modos de vida. }\end{array}$ \\
\hline Pertinencia & $\begin{array}{l}\text { La existencia de una base natural biodiversa en los territorios de sociedades } \\
\text { tradicionales, fundamenta la oferta de recursos naturales disponibles, a partir de } \\
\text { los cuales, se sostiene el sistema de autosuficiencia alimentaria de las familias. } \\
\text { Sobre estos inventarios de vida, las autoridades tradicionales ejercen una } \\
\text { gobernanza para garantizar el acceso y la sustentabilidad de los mismos. }\end{array}$ \\
\hline Unidad de medida & $\begin{array}{l}\text { Porcentaje (\%) de extensión en la cobertura (i), en territorios sociedades } \\
\text { tradicionales (j), en el año (k). }\end{array}$ \\
\hline
\end{tabular}

\begin{tabular}{|l|l|}
\hline \multicolumn{1}{|c|}{ Indicador } & \multicolumn{1}{|c|}{ Áreas que se superponen con territorios sociedades tradicionales } \\
\hline Definición & $\begin{array}{l}\text { Son aquellas áreas de protección, reserva forestal y/o político administrativas que } \\
\text { cuentan con un estado legal del territorio, las cuales se superponen con las áreas } \\
\text { de los territorios de sociedades tradicionales. }\end{array}$ \\
\hline Pertinencia & $\begin{array}{l}\text { El ordenamiento territorial, contempla diferentes formas del estado legal del } \\
\text { territorio; son las áreas de gobierno como reservas forestales óreas } \\
\text { infraestructura como hidroeléctricas y pozos extracción de hidrocarburos o para } \\
\text { cualquier otro uso, así como las zonas ilegales de la ganadería, la minería o la tala; } \\
\text { que se superponen con las comunidades tradicionales; generan conflictos } \\
\text { territoriales que afectan el control de estas personas sobre los recursos naturales, } \\
\text { o incluso deslegitiman a las autoridades locales en los territorios de sociedades } \\
\text { tradicionales; lo anterior, genera diferentes conflictos territoriales no resueltos que } \\
\text { afectan la gobernanza de los recursos naturales, que afectan los mismos en los } \\
\text { territorios de sociedades tradicionales y deslegitiman la autoridad tradicional. }\end{array}$ \\
\hline Unidad de medida & $\begin{array}{l}\text { Porcentaje (\%) de extensión de un área traslapada (i), en cada territorios } \\
\text { sociedades tradicionales (j), en el año (k). }\end{array}$ \\
\hline
\end{tabular}




\begin{tabular}{|l|l|}
\hline \multicolumn{1}{|c|}{ Indicador } & \multicolumn{1}{|c|}{ Potencial de tierra cultivables demandadas por unidades familiares } \\
\hline Definición & $\begin{array}{l}\text { Se trata de las áreas cultivadas por las unidades familiares, que se demanda en el } \\
\text { contexto de su sistema de producción y bajo las exigencias de la sostenibilidad de } \\
\text { sus modos de vida, en los territorios de sociedades tradicionales. }\end{array}$ \\
\hline Pertinencia & $\begin{array}{l}\text { El acceso a las tierras por parte de las unidades familiares es una garantía para el } \\
\text { sostenimiento de la autosuficiencia alimentaria. Permitirá ampliar los estudios } \\
\text { asociados a las unidades de producción familiar hechos de manera general, pero } \\
\text { que ameritan una especificación de acuerdo al sistema productivo de cada pueblo. }\end{array}$ \\
\hline Unidad de medida & $\begin{array}{l}\text { Porcentaje (\%) de extensión de áreas en chagras (i), en territorios sociedades } \\
\text { tradicionales (j), en el año (k). }\end{array}$ \\
\hline
\end{tabular}

\begin{tabular}{|l|l|}
\hline \multicolumn{1}{|c|}{ Indicador } & \multicolumn{1}{|c|}{ Potencial de población en territorios sociedades tradicionales } \\
\hline Definición & $\begin{array}{l}\text { Se refiere al estado de la población neta en un momento dado, como producto de } \\
\text { su dinamismo y en referencia a: nacimientos, defunciones y el saldo migratorio, } \\
\text { con respecto al total de población. }\end{array}$ \\
\hline Pertinencia & $\begin{array}{l}\text { Conocimiento sobre las dinámicas sociales que impactan el crecimiento y } \\
\text { composición (edad y sexo) de la población; el saldo entre fecundidad y la } \\
\text { mortalidad, muestra el estado de salud y las condiciones socioeconómicas de la } \\
\text { población. El saldo migratorio en los territorios de sociedades tradicionales, } \\
\text { permite conocer la movilidad de la población en el espacio y a través del tiempo; } \\
\text { permitirá comprender: las razones por las cuales la población migra; las razones e } \\
\text { impactos de orden social, sobre la demografía de la población, así como las } \\
\text { tendencias y las expectativas de los lugareños por los desplazamientos hacia los } \\
\text { territorios de sociedades tradicionales. }\end{array}$ \\
\hline Unidad de medida & $\begin{array}{l}\text { Total crecimiento natural }\left(\mathrm{N}^{\circ}\right) \text { población con dinámica (i), en cada territorio } \\
\text { colectivo (j), en el año }(\mathrm{k}) .\end{array}$ \\
\hline
\end{tabular}

\begin{tabular}{|l|l|}
\hline \multicolumn{1}{|c|}{ Indicador } & \multicolumn{1}{c|}{ Auto - reconocimiento étnico } \\
\hline Definición & $\begin{array}{l}\text { Expresa el tipo de identificación étnica que los individuos manifiestan pertenecer } \\
\text { en los territorios sociedades tradicionales; es decir el auto-reconocimiento dentro } \\
\text { de un grupo étnico particular. }\end{array}$ \\
\hline Pertinencia & $\begin{array}{l}\text { Conocimiento sobre los grupos étnicos existentes, a los cuales están adscritos los } \\
\text { habitantes en los territorios. El auto reconocimiento permite mostrar la cohesión } \\
\text { respecto a los grupos en los que se ejerce una u otra actividad cultural; igualmente, } \\
\text { el estado sobre la conciencia étnica vinculada directamente al ejercicio de sus } \\
\text { derechos colectivos como pueblos -autodeterminación-. }\end{array}$ \\
\hline Unidad de medida & $\begin{array}{l}\text { Porcentaje (\%) de población que se identifica con una etnia (i), en unos territorios } \\
\text { sociedades tradicionales (j), en un año (k). }\end{array}$ \\
\hline
\end{tabular}




\begin{tabular}{|l|l|}
\hline \multicolumn{1}{|c|}{ Indicador } & \multicolumn{1}{|c|}{ Participación de la población en prácticas culturales estratégicas } \\
\hline Definición & $\begin{array}{l}\text { Establecer la vigencia de las prácticas culturales en el contexto del siglo XXI a través } \\
\text { de la medición de la participación de la población en los eventos en que tienen } \\
\text { lugar. }\end{array}$ \\
\hline Pertinencia & $\begin{array}{l}\text { La medición de la vigencia de estas prácticas culturales permite dar cuenta de las } \\
\text { posibilidades de ejercer las actividades asociadas a la cultura que finalmente } \\
\text { determinan el status identitario y reafirman la particularidad y la diferencia de las } \\
\text { mismas. }\end{array}$ \\
\hline Unidad de medida & $\begin{array}{l}\text { Porcentaje (\%) de participación de la población en una práctica cultural (i), en un } \\
\text { territorios sociedades tradicionales (j), en un año (k). }\end{array}$ \\
\hline
\end{tabular}

\begin{tabular}{|l|l|}
\hline \multicolumn{1}{|c|}{ Indicador } & \multicolumn{1}{|c|}{ Personas bilingües indígenas -lengua propia - español- } \\
\hline Definición & $\begin{array}{l}\text { Se ocupa de medir el número de personas que es competente lingüísticamente en } \\
\text { la práctica de la lengua propia como en el español y/o portugués. }\end{array}$ \\
\hline Pertinencia & $\begin{array}{l}\text { Determinar la variabilidad de situaciones de bilingüismo, con diferentes grados de } \\
\text { asimetría y de intervención de las lenguas dominantes. La pertinencia cultural de } \\
\text { las políticas públicas para personas bilingües, estará dada por la articulación de las } \\
\text { especificidades culturales y la identidad local propia, rechazando la } \\
\text { homogeneización y la imposición de modelos occidentales globalizados. El } \\
\text { bilingüismo en idioma propio, español y/o portugués, debe considerarse como un } \\
\text { indicador de pluralismo y riqueza cultural y no como una barrera cultural o } \\
\text { subdesarrollo. }\end{array}$ \\
\hline Unidad de medida & $\begin{array}{l}\text { Porcentaje (\%) de personas de una población que son bilingües (i), en un territorios } \\
\text { sociedades tradicionales (j), en un año (k). }\end{array}$ \\
\hline
\end{tabular}

\begin{tabular}{|l|l|}
\hline \multicolumn{1}{|c|}{ Indicador } & \multicolumn{1}{|c|}{ Grado de educación en el sistema de educación formal } \\
\hline Definición & $\begin{array}{l}\text { Determina el porcentaje de personas que cuentan con años cursados y aprobados, } \\
\text { en educación primaria, secundaria, superior, tecnológica, en el sistema formal de } \\
\text { educación. }\end{array}$ \\
\hline Pertinencia & $\begin{array}{l}\text { El nivel alcanzado en el contexto de la educación formal, permite establecer las } \\
\text { posibilidades para interactuar con el sistema de valores no- indígenas para sostener } \\
\text { procesos interculturales; así como la influencia sobre el propio sistema de valores. }\end{array}$ \\
\hline Unidad de medida & $\begin{array}{l}\text { Porcentaje (\%) de personas que han cursado un grado escolar (i), en unos territorios } \\
\text { sociedades tradicionales (j), en un año (k). }\end{array}$ \\
\hline
\end{tabular}


Tabla 4. IBHI de la capacidad para garantizar la autonomía alimentaria

\begin{tabular}{|l|l|}
\hline \multicolumn{1}{|c|}{ Indicador } & \multicolumn{1}{|c|}{$\begin{array}{c}\text { Grado de diversidad de los alimentos producidos y consumidos por las } \\
\text { unidades familiares }\end{array}$} \\
\hline Definición & $\begin{array}{l}\text { Número de especies con sus variedades sembradas en los espacios cultivados, } \\
\text { recolectadas, pesca y cacería, por las unidades familiares de producción. }\end{array}$ \\
\hline Pertinencia & $\begin{array}{l}\text { La posibilidad de diversificación de los alimentos o de abastecerse con productos } \\
\text { de autosuficiencia alimentaria, otorga un grado mayor de bienestar; permite } \\
\text { establecer: derecho, la libertad, la emancipación, de los pueblos indígenas a } \\
\text { sembrar y producir los alimentos nutritivos y culturalmente adecuados, con sus } \\
\text { semillasy prácticas culturales propias de forma sostenible y ecológica, y su derecho } \\
\text { a decidir su propio sistema alimentario y productivo. }\end{array}$ \\
\hline Unidad de medida & $\begin{array}{l}\text { Porcentaje (\%) especies sembradas, recolectadas, pesca y cacería (i), en un } \\
\text { territorios sociedades tradicionales (j), en un año (k). }\end{array}$ \\
\hline
\end{tabular}

\begin{tabular}{|c|c|}
\hline Indicador & Importancia cultural de los alimentos de la dieta local \\
\hline Definición & $\begin{array}{l}\text { Importancia relativa de los alimentos en contextos locales a partir de criterios } \\
\text { culturales tales como: tabúes, motivaciones sociales colaterales, representaciones } \\
\text { sobre propiedades adicionales de tales productos, representaciones asociadas a la } \\
\text { identidad étnica, entre otros. }\end{array}$ \\
\hline Pertinencia & $\begin{array}{l}\text { Los medios a través de los cuales se logra la capacidad de alimentarse, son también } \\
\text { fundamentales a la hora de abastecerse, pues le permite una vinculación concreta } \\
\text { a esta sociedad. Las categorías de alimentos desde la perspectiva local, se } \\
\text { muestran no solo en términos del prestigio, sino también, de los tabúes y } \\
\text { prohibiciones que existen y que les permiten conservar un cierto estado de salud } \\
\text { bajo el respeto de las normas y prohibiciones derivadas de la tradición local y de la } \\
\text { noción de equilibrio energético. }\end{array}$ \\
\hline Unidad de medida & $\begin{array}{l}\text { Porcentaje }(\%) \text { tabúes y prohibiciones sobre un alimento (i), en un territorios } \\
\text { sociedades tradicionales (j), en un año }(k) \text {. }\end{array}$ \\
\hline
\end{tabular}

\begin{tabular}{|l|l|}
\hline \multicolumn{1}{|c|}{ Indicador } & \multicolumn{1}{|c|}{ Medios para la conservación y transformación de alimentos } \\
\hline Definición & $\begin{array}{l}\text { Existencia de formas y medios para el almacenamiento de ciertos productos de } \\
\text { autosuficiencia alimentaria, para su aprovechamiento en el largo plazo. }\end{array}$ \\
\hline Pertinencia & $\begin{array}{l}\text { La posibilidad de almacenar y/o conservar productos y semillas, son formas de } \\
\text { seguridad alimentaria. Estudios adelantados con pueblos indígenas en el } \\
\text { departamento del Amazonas, han demostrado que la conservación de biomasas } \\
\text { de yuca, de semillas almacenadas en las chagras, cocinas o en las orillas de los ríos, } \\
\text { garantiza un abastecimiento continuo, aún si existen situaciones climáticas } \\
\text { extremas e inundaciones de las tierras cultivables. }\end{array}$ \\
\hline
\end{tabular}




\begin{tabular}{|l|l|}
\hline Unidad de medida & $\begin{array}{l}\text { Número }\left(\mathrm{N}^{\circ}\right) \text { de formas de almacenamiento y conservación de alimentos (i), en un } \\
\text { territorios sociedades tradicionales }(\mathrm{j}) \text {, en un año }(\mathrm{k}) .\end{array}$
\end{tabular}

\begin{tabular}{|l|l|}
\hline Indicador & \multicolumn{1}{|c|}{$\begin{array}{c}\text { Disponibilidad de semillas tradicionales e introducidas en las unidades } \\
\text { familiares }\end{array}$} \\
\hline Definición & $\begin{array}{l}\text { Inventarios de las semillas fundamentales para garantizar la siembra de los } \\
\text { espacios cultivados, cuya producción permite un abastecimiento continuo, para } \\
\text { garantizar no solo la abundancia de alimentos, sino la reproducción cultural. }\end{array}$ \\
\hline Pertinencia & $\begin{array}{l}\text { La producción permanente de los espacios cultivados establecidos por las familias, } \\
\text { es fundamental para garantizar la autosuficiencia alimentaria; las familias deben } \\
\text { contar la capacidad de un inventario de semillas que permitan sostener el ciclo } \\
\text { agrícola sustentado en la apertura, siembra, mantenimiento y aprovechamiento de } \\
\text { los espacios cultivados. }\end{array}$ \\
\hline Unidad de medida & $\begin{array}{l}\left.\text { Número ( }{ }^{\circ}\right) \text { de semillas por especie disponibles para la siembra (i), en un } \\
\text { territorios sociedades tradicionales (j), en un año (k) }\end{array}$ \\
\hline
\end{tabular}

\begin{tabular}{|l|l|}
\hline \multicolumn{1}{|c|}{ Indicador } & \multicolumn{1}{c|}{ Población con desnutrición } \\
\hline Definición & $\begin{array}{l}\text { Determina el porcentaje de personas que registran problemas de desnutrición, por } \\
\text { deficiencias en la ingesta de calorías y proteínas, ocasionadas por el limitado } \\
\text { acceso a los alimentos; genera repercusiones no solo en el estado de la salud de la } \\
\text { población sino los diferentes efectos sociales. }\end{array}$ \\
\hline Pertinencia & $\begin{array}{l}\text { La nutrición de los individuos permite dar cuenta de las posibilidades reales de } \\
\text { consumo de alimentos. La valoración del estado nutricional, es un parámetro que } \\
\text { permite evaluar el estado de la salud de la población y sus diferentes incidencias } \\
\text { sociales, con los niveles de seguridad alimentaria de la población, la cual a su vez } \\
\text { está determinada por múltiples factores que inciden en el acceso a los alimentos. }\end{array}$ \\
\hline Unidad de medida & $\begin{array}{l}\text { Porcentaje (\%) de personas con desnutrición (i), en territorios de sociedades } \\
\text { tradicionales (j), en un año (k). }\end{array}$ \\
\hline
\end{tabular}

Tabla 5. IBHI de la capacidad para lograr un ambiente tranquilo

\begin{tabular}{|l|l|}
\hline \multicolumn{1}{|c|}{ Indicador } & \multicolumn{1}{c|}{ Ejercicio de la territorialidad } \\
\hline Definición & $\begin{array}{l}\text { Son las relaciones que se construyen en el territorio, entendidas como un } \\
\text { entramado de relaciones sociales que supera ampliamente la visión puramente } \\
\text { espacial del mismo, a un concepto sobre la territorialidad, como un sistema de } \\
\text { conductas que controlan y mantienen un uso y manejo específicos sobre un } \\
\text { espacio. }\end{array}$ \\
\hline Pertinencia & $\begin{array}{l}\text { El conocimiento sobre los territorios de sociedades tradicionales, el tipo y estado } \\
\text { de las relaciones tanto internas como hacia el exterior, exige su análisis de manera } \\
\text { que puedan ser tipificadas e instrumentalizadas; o sea, el sistema de conductas que }\end{array}$ \\
\hline
\end{tabular}




\begin{tabular}{|l|l|}
\hline & $\begin{array}{l}\text { controlan y mantienen un uso y manejo específicos sobre un territorio. Ser } \\
\text { autónomo respecto a agentes y problemáticas sociales externas para el ejercicio } \\
\text { de la territorialidad, implica dar cuenta de actores externos que generan tensiones } \\
\text { en los territorios de sociedades tradicionales. }\end{array}$ \\
\hline Unidad de medida & $\begin{array}{l}\text { Porcentaje (\%) de conflictos internos y externos existentes (i), en un territorios } \\
\text { sociedades tradicionales (j), en un año (k). }\end{array}$ \\
\hline
\end{tabular}

Tabla 6. IBHI de la capacidad para asegurar el auto-cuidado y la reproducción

\begin{tabular}{|l|l|}
\hline \multicolumn{1}{|c|}{ Indicador } & \multicolumn{1}{|c|}{ Acceso a servicios públicos básicos en territorios sociedades tradicionales } \\
\hline Definición & $\begin{array}{l}\text { Establece la proporción de hogares que tienen acceso a los servicios básicos } \\
\text { públicos (agua potable, alcantarillado, recolección de basuras y energía eléctrica). }\end{array}$ \\
\hline Pertinencia & $\begin{array}{l}\text { La identificación de los servicios públicos existentes como: agua potable, } \\
\text { disposición final de basuras, alcantarillado, energía eléctrica; servicios básicos que } \\
\text { permiten mejores condiciones de salubridad y bienestar de las familias en las } \\
\text { comunidades indígenas. }\end{array}$ \\
\hline Unidad de medida & $\begin{array}{l}\text { Porcentaje (\%) de población con cobertura de servicios públicos básico (i), en un } \\
\text { territorios sociedades tradicionales }(\mathrm{j}) \text {, en un año (k). }\end{array}$ \\
\hline
\end{tabular}

\begin{tabular}{|l|l|}
\hline \multicolumn{1}{|c|}{ Indicador } & \multicolumn{1}{|c|}{ Casos de personas evitables por alternativas tradicionales } \\
\hline Definición & $\begin{array}{l}\text { Métodos terapéuticos existentes para la prevención de la salud física y espiritual, } \\
\text { tanto por servicios formales prestados por el Estado, como a través de la medicina } \\
\text { tradicional, con base en el respeto de normas y valores dados culturalmente. }\end{array}$ \\
\hline Pertinencia & $\begin{array}{l}\text { Establecer el estado de los métodos terapéuticos y preventivos que existen y los } \\
\text { agentes quienes regularmente atiende los casos de enfermedades en las } \\
\text { comunidades indígenas; el tipo de prácticas, rituales, recursos, que se utilizan para } \\
\text { prevención de las enfermedades; y los desafíos para evitar las principales } \\
\text { enfermedades. }\end{array}$ \\
\hline Unidad de medida & $\begin{array}{l}\text { Porcentaje (5) de servicios de prevención de la salud física y espiritual existe (i), en } \\
\text { un territorios sociedades tradicionales (j), en un año (k). }\end{array}$ \\
\hline
\end{tabular}

\begin{tabular}{|l|l|}
\hline \multicolumn{1}{|c|}{ Indicador } & Cobertura de los medios para atender enfermedades sociedades tradicionales \\
\hline Definición & $\begin{array}{l}\text { Acceso a servicios de salud proveídos por: el Estado -atención primaria, medicina } \\
\text { farmacéutica, intervención hospitalaria, sistemas de inmunización-; servicios de } \\
\text { medicina tradicional. }\end{array}$ \\
\hline Pertinencia & $\begin{array}{l}\text { Establece el acceso a los servicios brindados por los sistemas de salud existentes en } \\
\text { las comunidades indígenas. Conocer los servicios que presta el sistema de salud } \\
\text { formal y los servicios que presta la medicina tradicional; permite esclarecer las }\end{array}$ \\
\hline
\end{tabular}




\begin{tabular}{|l|l|}
\hline & $\begin{array}{l}\text { condiciones sociales existentes en las que es posible y deseable el desarrollo de la } \\
\text { vida en las comunidades. }\end{array}$ \\
\hline Unidad de medida & $\begin{array}{l}\text { Porcentaje (\%) población atendida por un servicio de salud disponible (i), en un } \\
\text { territorios sociedades tradicionales (j), en un año }(\mathrm{k}) .\end{array}$ \\
\hline
\end{tabular}

\begin{tabular}{|l|l|}
\hline \multicolumn{1}{|c|}{ Indicador } & \multicolumn{1}{c|}{ Atención de mujeres en parto } \\
\hline Definición & $\begin{array}{l}\text { Son las posibilidades que existen de lograr un parto asistido de manera eficiente, } \\
\text { sea por médicos tradicionales o por el sistema de atención de salud pública. }\end{array}$ \\
\hline Pertinencia & $\begin{array}{l}\text { El número de partos atendidos de manera efectiva es un indicador de salud, ya que } \\
\text { su atención oportuna atenúa de manera significativa la morbilidad y la mortalidad } \\
\text { materna y perinatal. Busca además, dar cuenta de las debilidades de la atención de } \\
\text { la salud en los territorios sociedades tradicionales. }\end{array}$ \\
\hline Unidad de medida & $\begin{array}{l}\text { Porcentaje (\%) de partos asistidos por servicios médicos tradicionales y/o formales } \\
\text { (i), en el territorios sociedades tradicionales (j), en un año (k). }\end{array}$ \\
\hline
\end{tabular}

\begin{tabular}{|l|l|}
\hline \multicolumn{1}{|c|}{ Indicador } & \multicolumn{1}{|c|}{ Practica de disciplinas deportivas en territorios de sociedades tradicionales } \\
\hline Definición & $\begin{array}{l}\text { Se refiere a la participación de la sociedad en los deportes tradicionales en el } \\
\text { carácter oficial de los hechos en los territorios tradicionales }\end{array}$ \\
\hline Pertinencia & $\begin{array}{l}\text { Los deportes tradicionales son importantes para el desarrollo físico con identidad. } \\
\text { Conocer el nivel de la práctica de estas disciplinas y discapacidades del desarrollo, } \\
\text { contribuye a la resistencia cultural a partir de este punto de vista, y al mismo tiempo } \\
\text { se promueve como una alternativa fundamental para llenar el tiempo libre de una } \\
\text { manera saludable, especialmente en los jóvenes }\end{array}$ \\
\hline Unidad de medida & $\begin{array}{l}\text { Porcentaje (\%) de deportes tradicionales practicados (i), en el territorios } \\
\text { sociedades tradicionales (j), en un año (k). }\end{array}$ \\
\hline
\end{tabular}

Fuente. Elaboración propia

En el año 2016, fruto de un proceso de cooperación científica internacional entre el Grupo de Investigación Educación y Diversidad del Amazonas de la Universidad del Estado de Amazonas (UEA GPEDA-) y el Grupo de Investigación Valoración de los Conocimientos Tradicionales, del Instituto Amazónico de Investigaciones Científicas, Sinchi, de Colombia. Los IBPT fueron aplicados tres comunidades indígenas e ribereñas, en el municipio de Benjamín Constant, en la amazonia brasilera, en la zona de triple frontera que comparten Brasil, Colombia y Perú: comunidad Sao Joao de Veneza (etnia Ticuna), comunidad Nova 
Alianca (etnia Cocama), comunidad de Sao José. Los resultados reportados por Acosta y Lacerda (2016), muestran que el bienestar para estas comunidades indígenas y ribereñas reside en contar con un buen gobierno que garantice la abundancia de alimentación y el cumplimiento de las normas comunitarias, en armonía entre las diferentes tendencias culturales y religiosos en la perspectiva de un lugar común. Es la base de la cohesión, la cooperación y la solidaridad de la comunidad que generan buenas condiciones de vida.

Dichos autores concluyen que la aplicación de los IBPT muestra importantes avances metodológicos en la construcción de indicadores fiables sobre el bienestar de las poblaciones tradicionales e ilustran cómo las comunidades tradicionales construyen su bienestar mediante la sabiduría sobre los ciclos de agua, la tierra y el bosque, manteniendo la autonomía sobre procesos productivos y culturales en sus territorios, como una forma de equilibrar la tradición y la innovación que viene de los tiempos actuales.

\section{Conclusiones}

El paradigma del cambio climático viene ampliándose y profundizándose en razón a los aportes de diferentes evaluaciones y pronunciamientos a nivel planetario. Recientemente se sostiene que el cambio climático multiplicará las amenazas que podrían generar situaciones de inseguridad hídrica, energética, alimentaria y de salud, en razón a la tendencia que muestra la intensificación de los impactos por los desastres naturales generados por la vigencia del modelo económico de crecimiento y las mayores intervenciones humanas sobre el medio ambiente; se argumenta que la sostenibilidad de los ecosistemas en la Amazonia, son una seguridad para mantener el equilibrio en el clima a nivel planetario y que los recursos naturales son una seguridad no solo ambiental, sino que garantizan el bienestar de las poblaciones y la economía en la región.

Entender las causas y las consecuencias del cambio del clima en los ecosistemas y en las sociedades, exige contar con otros enfoques multidimensionales, en razón a las particularidades culturales y cosmogónicas, de las sociedades tradicionales que habitan los ecosistemas en la Amazonia. Los modelos para la identificación y diseño de indicadores apropiados para sociedades tradicionales, han tenido en cuenta los aportes de entidades y organismos de orden internacional, y su preocupación en la búsqueda de indicadores no convencionales referidos a evaluar los alcances de las políticas de desarrollo y el 
reconocimiento de los derechos humanos. El modelo de capacidades y los IBPT aplicados en la Amazonia colombiana y brasilera, los resultados alcanzados muestran que se trata de una herramienta útil y a disposición de las autoridades tradicionales; les permita contar con la información necesaria que los faculte efectuar las evaluaciones periódicas sobre sus modos de vida y bienestar humano en sus territorios y su relación con la sostenibilidad de la naturaleza. Además, ofrecer la posibilidad de visibilizar las particularidades y problemáticas del contexto ambiental, social, económico, cultural y político, donde se desenvuelven sus modos de vida. La aplicación de los IBPT adelantada en la Amazonia colombiana y brasilera, y fruto de la cooperación internacional entre la Universidad del Estado de Amazonas (UEA) y el Instituto Amazónico de Investigaciones Científicas, Sinchi, reconocen que las cosmovisiones que se construyen y reproducen las sociedades tradicionales desde lo local, son fundamentales para evaluar tanto las políticas como las acciones de desarrollo que se dirigen a asimilar a las sociedades indígenas a los rigores de la economía de mercado.

La aplicación de los IBPT provee un marco conceptual y metodológico, cuya información obtenida periódicamente con la participación directa y comprometida de las sociedades tradicionales, son una innovación social con el potencial de contribuir a retroalimentar la formulación de políticas públicas, programas y ejecución de proyectos que promuevan el desarrollo y el bienestar humano en los territorios de las sociedades tradicionales; en particular es un modelo multidimensional con la capacidad de contar con información que permita anticipar y atenuar los rigores de los impactos del cambio climático.

\section{Bibliografía}

Acosta, L.E. (2013). Pueblos indígenas de la Amazonia e indicadores de desarrollo humano sostenible en la encrucijada de la globalización: estudio de caso Amazonia Colombiana. Tesis Doctoral: Doctorado Globalización, Desarrollo y Cooperación Internacional, de la Facultad de Ciencias Económicas y Empresariales (Departamento de Economía Aplicada I). Universidad del País Vasco. Instituto Amazónico de Investigaciones Científicas - Sinchi. Bilbao: p. 329.

Acosta, L.E., y Mendoza, D. (2006). El conocimiento tradicional: clave en la construcción del desarrollo sostenible en la amazonia colombiana. Revista Colombia Amazónica., Edición Es, pp.101-118

Acosta, L.E., Pérez, M., Juragaro, L., Nonokudo, H., Sánchez, G., Zafiama, Á., Tejada, J., Cobete, O., Efaiteke, M., Farekade, J., Giagrekudo, H., y Neikase, S. (2011). La chagra en La Chorrera: más que una producción de subsistencia, es una fuente de 
comunicación y alimento físico y espiritual, de los Hijos del Tabaco, la Coca y la Yuca dulce. Los retos de las nuevas generaciones para las prácticas culturales y los saberes tradicionales asociados a la biodiversidad. Instituto Amazónico de Investigaciones Científicas, Sinchi. Asociación Zonal Indígena de Cabildos y Autoridades Tradicionales de La Chorrera - AZICATCH. Leticia: p. 136.

Acosta, L.E., Garcia, O.I., Dubois, A. (2016). Las capacidades colectivas como un instrumento metodológico para la evaluación del bienestar humano en territorios indígenas del Amazonas colombiano. Revista Mundo Amazónico. Universidad Nacional de Colombia. (Aprobado para publicación). Bogotá.

http://www.revistas.unal.edu.co/index.php/imanimundo

Acosta, L.E., Lacerda, L.F. (2016). Indicadores de Bem-Estar Humano para Povos Tradicionais: O caso de uma comunidade ribeirinha na fronteira da Amazônia brasileira". En: Revista Ciências Sociais de la Universidade Sinos (Brasil). Instituto Sinchi. Leticia. $30 \mathrm{p}$. http://revistas.unisinos.br/index.php/ciencias_sociais/author/submission/11734

Bergkamp, G., Orlando, B. and Burton, I. (2003). Adaptation of water management to climate change. IUCN, Gland, Switzerland and Cambridge, UK. Traducción: José María Blanch.

Centro Latinoamericano y Caribeño de Demografía - CELADE / Comisión Económica para América Latina y el Caribe - CEPAL - Fondo Indígena. (2007). Sistema de Indicadores Sociodemográfico de Poblaciones y Pueblos Indígenas de América Latina - SISPPI. Guía para el usuario. Del Popolo, F; Oyarce, AM; Ribotta. En: http://celade.cepal.org/redatam/PRYESP/SISPPI/SISPPI_notastecnicas.pdf (Consultada: 3 de mayo de 2011)

Dubois, A. (2008). El debate sobre el enfoque de las capacidades: las capacidades colectivas. Araucaria Revista Iberoamericana de Filosofía, Política y Humanidades, № 20 Segundo semestre de 2008. Pág. 35 -63. http://institucional.us.es/araucaria/nro20/nro20.htm (Consultada: 17 de julio de 2012)

De La Cruz Nassar, P.E. (2015). Ferias de Chagras en la Amazonia colombiana, contribuciones a los conocimientos tradicionales, y al intercambio de productos de las asociaciones indígenas y de mujeres de Tarapacá. El Colegio de la Frontera Sur ECOSUR.

De la Cruz, PE. (2016). Proyecto de investigación de la Tesis de Doctorado: "La valoración de los conocimientos tradicionales en la construcción del territorio en la Amazonía colombiana", programa de doctorado Ciencias en Ecología y Desarrollo Sustentable en el Colegio de la Frontera Sur - ECOSUR. Instituto Sinchi. Chiapas, México.

Elías, S; Cardona, J. (2015). Conocimientos Tradicionales para la Adaptación al Cambio Climático en el Altiplano Occidental de Guatemala. The Nature Conservancy. Agencia de los Estados Unidos para el Desarrollo Internacional (USAID). para la Adaptación al Cambio Climático en el Altiplano Occidental de Guatemala. 
Guatemala. 72 p. http://www.usaid-cncg.org/wpcontent/uploads/2015/03/Conocimientos_tradicionales_ccl_final.pdf

Feldt, H. (2011). Fortalecimiento de Organizaciones Indígenas en América Latina: Pueblos Indígenas y Cambio Climático. Relación entre cambio climático y pueblos indígenas y sus posiciones en el contexto de las negociaciones en la Convención Marco sobre el Cambio Climático. GIZ 18 p. http://www.giz.de

Garcia, O.I. (2009). Analyse ethnographique d'un processus d'éthnodéveloppement dans l'Amazonie colombienne: «Les Fils du Tabac, de la Coca et du Manioc doux». Tesis de Maestría. EHESS. Paris

Gasché, J., Vela, N. (2004). Estudio de incentivos para conservación y uso sostenible de la biodiversidad en bosques de comunidades bosquesinas. Instituto Investigaciones de la Amazonia Peruana - IIAP. CONCYTEC. 56 p.

Gasché. J. (2012). Sociedad Bosquesina: Tomo I. Instituto de Investigaciones de la Amazonia Peruana. Iquitos- Peru.

Grupo Semillas (2014); ¿Cambio climático o vulnerabilidad del territorio? Revista Semillas N 57 y 50. Bogotá. 102 p. www.semillas.org.co

IPCC. (2014). Cambio climático 2014: Impactos, adaptación y vulnerabilidad - Resumen para responsables de políticas. Contribución del Grupo de trabajo II al Quinto Informe de Evaluación del Grupo Intergubernamental de Expertos sobre el Cambio Climático [Field, C.B., V.R. Barros, D.J. Dokken, K.J. Mach, M.D. Mastrandrea, T.E. Bilir, M. Chatterjee, K.L. Ebi, Y.O. Estrada, R.C. Genova, B. Girma, E.S. Kissel, A.N. Levy, S. MacCracken, P.R. Mastrandrea y L.L. White (eds.)]. Organización Meteorológica Mundial, Ginebra, Suiza, 34 págs. www.ipcc.ch.

Lacerda, L. F. (2016). Por uma sociologia das emergências: perspectivas emancipatórias nos territórios de produção das ausências amazônicas. São Leopoldo- RS, Tese de Doutorado. Universidade do Vale do Rio dos Sinos, 396p.

Mardas, N., Bellfield, H., jarvis, A., Navarrete, C., Comberti, C. (2013). Agenda de seguridad para la Amazonia: resumen de hallasgos y recomendaciones iniciales. Global Conopy Programme y Internationa Center for Tropical Agriculture. 23 p.

Organización Internacional del Trabajo - OIT. (2009). Los derechos de los pueblos indígenas y tribales en la práctica. Una guía sobre el Convenio $\mathrm{N}^{\circ} 169$ de la OIT. Programa para promover el Convenio $N^{\circ} 169$ de la OIT (pro 169). Departamento de Normas Internacionales del Trabajo. 201 p. Recuperado de http://www.ilo.org/wcmsp5/groups/public/@ed_norm/@normes/documents/publica tion/wcms_113014.pdf.

Organización de las Naciones Unidas - ONU. (2007). Declaración de las Naciones Unidas sobre los derechos de los pueblos indígenas. Resolución 61/295 aprobada por la Asamblea General. 107a. Sesión plenaria. Washington, DC. 
Organización de las Naciones Unidas - ONU. (2008). Los pueblos indígenas y los indicadores de bienestar y desarrollo. Informe Preliminar. Documento de trabajo. Recuperado de http://www.nacionmulticultural.unam.mx/Portal/Central/EDITORIAL/pdfs/080422_o nu_informe-es.pdf

Pardo, M. (2007). Energía y Sociedad: la transición energética hacia las energías renovables, SISTEMA: Revista de Ciencias Sociales, nº 162-163: 173-188.

Programa de las Naciones Unidas para el Medio Ambiente (PNUMA). 2009. Informe: Perspectivas del Medio Ambiente en la Amazonía - GEO Amazonía. Organización del Tratado de Cooperación Amazónica (OTCA). Brasília, Brasil. 168 p.

UNDP-DHA. (1994). An Overview of Disaster Management, Disaster Management Training Programme, Nueva York, $2^{\mathrm{a}}$ ed.

Ulloa, A.; Escobar, E.M., Donato, L.M., Escobar, P. (Hrsg.). (2008). Mujeres indígenas y cambio climático. Perspectivas latinoamericanas. Fundación Natura, Bogotá. Disponible en: file:///C:/Users/lacosta/Downloads/mujeres-indigenas-y-cambioclimatico-2008.pdf

Uribe, E. (2015). "El cambio climático y sus efectos en la biodiversidad en América Latina". Comisión Económica para América Latina y el Caribe (CEPAL). Unión Europea Programa EUROCLIMA (CEC/14/001). Santiago de Chile. 86 p. http://repositorio.cepal.org/bitstream/handle/11362/39855/S1501295_en.pdf;jsessio $\underline{\text { nid }=75 E 80796 B_{72} F_{3} 8040 C_{9} C_{49} 72 E B E C D B 1 A}$ ? sequence $=1$

\section{Resumo}

Este artigo expõe os antecedentes que é agora conhecido como o paradigma dos impactos das mudanças climáticas nos sistemas naturais e humanos; em especial, a gravidade dos seus impactos na Amazônia; o papel do conhecimento tradicional associado à biodiversidade, as suas experiências e cosmogonia, como uma alternativa não só para entender os rigores das alterações climáticas, mas as experiências podem ser retomadas como propostas destinadas a atenuar as alterações climáticas. Apresenta uma proposta que visa a obtenção de informações na Amazônia, através do qual ele se destina a ajudar a expandir as avaliações dos impactos das mudanças climáticas nas estruturas sociais com uma abordagem metodológica diferencial.

Palavras-chave: Mudança climática. Indicadores. Povos tradicionais. Indígenas - Amazônia. 